\title{
Development of EEG Data-driven Generative Art Application for Real-time and Dynamic Interaction
}

\author{
Chien-Tung Lin ${ }^{1}$, R.P.C. Janaka Rajapakse ${ }^{1, *}$, Yoshimasa Tokuyama ${ }^{2}$ \\ ${ }^{1}$ Graduate Institute of Animation and Film Art, Tainan National University of the Arts, No. 66, Daqi, Guantian Dist., Tainan City 72045, Taiwan \\ ${ }^{2}$ Department of Media and Image Technology, Tokyo Polytechnic University, 1583, Iiyama, Kanagawa 243-0297, Japan
}

\author{
ARTICLE INFO \\ Article History \\ Received 04 December 2020 \\ Accepted 07 May 2021 \\ Keywords \\ EEG \\ phyllotaxis \\ generative art \\ interactive art \\ installation art
}

\begin{abstract}
Generative art is produced by procedural techniques. It has obtained a lot of attention since the beginning of computer graphics. Many works of art are inspired by nature, among which phyllotaxis is as well. It is a combination of mathematics and the beauty of nature. Not only can it be seen everywhere in nature, but also often appear in man-made objects, becoming part of culture or religion. This paper presents the development of an interactive generative art application that is created from a phyllotaxis pattern by using the user's Electroencephalogram (EEG) data. When people are using it, it will allow them to more easily relax and achieve the function of art therapy. We tried to use EEG data to make an interactive installation art that creates phyllotaxis patterns that are projected on the wall. Everyone has a different state, the generated patterns are also different from person to person, which creates interesting interactive contents. In addition, sound can also be changed by EEG data to become dynamic and real-time contents.
\end{abstract}

(C) 2021 The Authors. Published by Atlantis Press International B.V.

This is an open access article distributed under the CC BY-NC 4.0 license (http://creativecommons.org/licenses/by-nc/4.0/).

\section{INTRODUCTION}

With the development of microcomputers and single chips, the way people interact with computers has changed. It has come a long way from the early keyboard and mouse to the development of the multi-touch. Developers always want to find better ways to let people interact with apps in a more natural way, such as through gestures (Leap Motion [1]), Wii [2], voice input, etc. However, these are all motor functions, that is, different people using the same operation method will get the same result. Compared with Electroencephalogram (EEG), because people cannot see how the user moves, it just exists in the brain's operation, so no one can copy the actions of another person and produce a personal style. Therefore, we aim at the state of concentration and meditation in the brain waves as an interactive input method to bring a new perspective.

In addition, installation art is becoming more and more popular as a form of modern art. Compared with other art forms, installation art allows people to be more involved, use touch, and even play in it. Therefore, interactive methods are also added to allow people to enjoy, participate in, communicate, and interact with art, making it truly interactive installation art. However, most of the art content is fixed or arranged according to the creator. So, there is not much change, resulting in people not wanting to repeat, come, and participate.

Generative art is produced by computer programs. It has the characteristics of non-repetition and endless evolution. It has received

"Corresponding author. Email: janakaraja@gmail.com a lot of attention since the beginning of computer graphics. Many works of art are inspired by nature, among which phyllotaxis is included. It is a combination of mathematics and the beauty of nature. Not only can it be seen everywhere in nature, but also often appear in man-made objects, becoming part of culture or religion. For example, Mandala Kolam is not only an expression of art but also healing people's bodies and minds. And Chakra's representative totem is also like phyllotaxis. When people are using it, it is easier to relax the mood and achieve the function of art therapy.

Therefore, we try to use EEG to make interactive installation art and use EEG input to create phyllotaxis patterns to project it on the wall. Because everyone has a different state, the graphics drawn are also different from person to person, creating interesting interactive works. In addition, the sound can also be changed by EEG, becoming a dynamic, real time content.

Finally, when the user looks at the graphics generated by his own brain, the visual and sound effects of these graphics can trigger psychological states to achieve an increasingly relaxed state, and achieve the effect of meditation. This can bring about communication with the heart and then achieve the effect of art therapy [3].

\section{BACKGROUND: EEG CONTROL ART}

\subsection{The Use of EEG in Art}

Since 1965, the use of EEG in artistic creation has been documented. Most of the content has an established pattern. Authors or users use their brains or mental states as artistic creations, such as 
focusing or relaxing to control sound and images [4]. That is active input agency.

There are three agencies for the Brain-Computer Interfaces (BCI). The active input agency, the reactive input agency, and the passive input agency. The reactive input agency is that the user's brain activity is changed by an external stimulus. Therefore, the training is needed for these two kinds of BCI [4].

\subsection{Passive Input Agency and Aesthetics}

Unlike the active input, the passive input agency does not require the participants to do special things with EEG as artistic creation. It uses the mental and emotional state of the author or participant to produce changes in sound and images, especially music that affects the emotional changes of the user. The changing screen is synchronized with the music [5]. In this application, the sound is also generated from the real-time EEG data which makes it easier for participants to immerse themselves in an atmosphere of discord.

However, this application goes beyond this way of expression and focuses more on the communication between the self and the heart. Through the displayed graphics and sounds to perceive and explore one's mental state, we can better open up internal communication and dialogue to achieve relaxation and meditation. This allows for self-reflection, and ultimately the opportunity to achieve the goal of art therapy.

\section{DEVELOPMENT AND APPLICATION}

\subsection{System Structure}

The development environment is Intel I7 PC on windows 10 system, with the NVIDIA GeForce RTX 2080TI graphics card. The programming language $\mathrm{C \#}$ has been used to develop the application on the Unity SDK. The EEG headset is NeuroSky MindWave Mobile 2.

Figures 1 and 2 show the EEG headset is connected to the computer via Bluetooth, and uses the intermediary software ThinkGear Connector [6] to send the received data to Unity [7] via UDP network protocol.

In NeuroSky EEG, there is a technology called ThinkGear, whose architecture is a sensor that touches the forehead, the contact points and reference points on the earpads, and the onboard chip for processing all data. The ThinkGear chip analyzes, calculates, and outputs the brainwave data, including the Attention and Meditation measures [8].

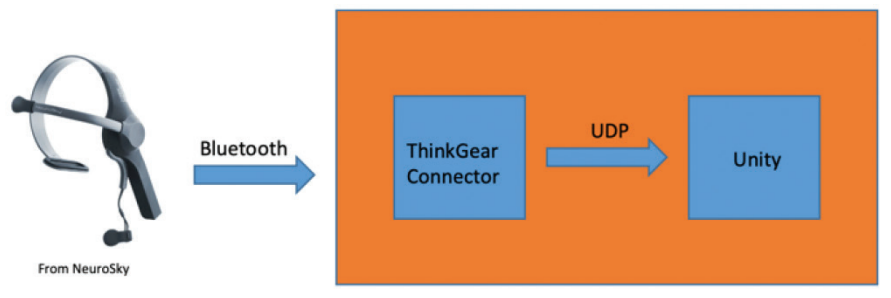

Figure 1 EEG connecting to Unity by Bluetooth and UDP.

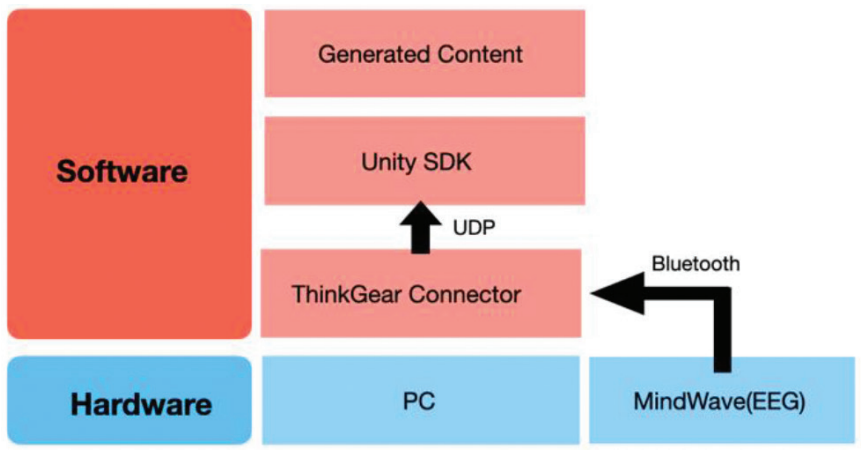

Figure 2 System structure.

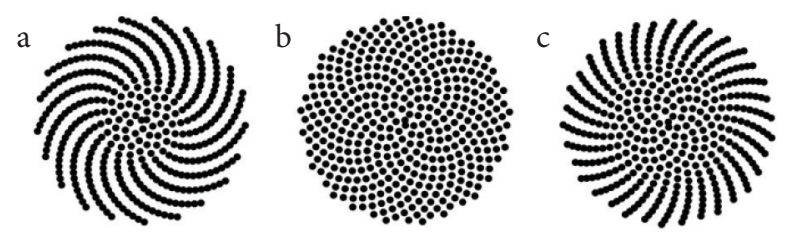

Figure 3 Generating phyllotactic patterns from the Vogel's formula. (a) $\alpha=137.3^{\circ}$, (b) $\alpha=137.5^{\circ}$, (c) $\alpha=137.6^{\circ}$.

\subsection{Content Generation}

The simple model for the florets of a sunflower was Vogel 's formula. As the book "The Algorithmic Beauty of Plants" [9] descript below:

$$
\varphi=n * 137.5^{\circ}, \quad r=c \sqrt{n},
$$

- $n$ is the ordering index number of a floret, from the center to outer. Therefore, it is accumulated from the minimum value to the maximum value in the program.

- $\varphi$ is the angle of the $n$th floret in a polar coordinate system.

- $r$ is the distance from the center of the capitulum to the $n$th floret.

- $c$ is a constant scaling.

- The number 137.5 is the angle of this pattern. In the application, the pattern is changed by this parameter. Therefore, it is not constant 137.5.

From this equation, we can get $r, \varphi$. From $r, \varphi$ we can calculate the $x, y$ values of the coordinate system to draw the pattern.

$$
x=r * \cos (\varphi), \quad y=r * \sin (\varphi) .
$$

In Figure 3, it can be found that changing different angles will produce different pattern changes, so the angle is used as a variable in the program to generate changeable patterns.

\subsection{EEG Data}

Different waveform data can be obtained from the signal from the brain waves, such as alpha wave, beta wave, etc., as well as data on concentration and meditation. We used the level of concentration to generate graphics, and use the level of meditation as a reference value for color and sound. 
In Table 1, the Attention value is reported on a relative eSense scale of $1-100$ [10]. It is direct-mapped into seven ranges to change the angle of the pattern. When the value of attention is low, the angle of the pattern is smaller; when the value is higher, the angle is larger.

Similarly, the Meditation value is reported on a relative eSense scale of 1-100 [10]. It is direct-mapped into seven ranges to change the color of the pattern as shown in Table 2. They are represented by seven colors of red, orange, yellow, green, blue, indigo, and violet. Obviously, these colors are the same as the chakra colors [11] , and they also form the basic color of white light. This correspondence is natural and conforms to the human body.

Figure 4 shows the patterns change depending on the value of the seven levels. The color of the pattern is changed from warm color to cold color. The user also becomes calmer and gradually enters the deep state of meditation.

In the sound part, the sound of ocean waves is used in the background, which makes people feel relaxed. In addition, different frequencies of Tibetan bowl sounds are played by EEG's meditation state. When the participant enters deep meditation, the system will play a calmer bowling sound.

Table 1 Use value of attention for angle $\varphi$

\begin{tabular}{lc}
\hline Range of attention & Angle $\boldsymbol{\varphi}$ \\
\hline $0-14$ & 41 \\
$15-29$ & 59 \\
$30-44$ & 73 \\
$45-59$ & 97 \\
$60-74$ & 109 \\
$75-89$ & 137.5 \\
$90-100$ & 157 \\
\hline
\end{tabular}

Table 2 Use value of meditation for color

\begin{tabular}{ll}
\hline Range of meditation & Color \\
\hline $0-14$ & RED \\
$15-29$ & ORANGE \\
$30-44$ & YELLOW \\
$45-59$ & GREEN \\
$60-74$ & BLUE \\
$75-89$ & INDIGO \\
$90-100$ & VIOLET \\
\hline
\end{tabular}

\begin{tabular}{|c|c|c|c|c|}
\hline Angle & 41 & 59 & 73 & 97 \\
\hline Color & Red & Orange & Yellow & Green \\
\hline Pattern & & & & \\
\hline Angle & 109 & 137.5 & 157 & \\
\hline Color & Blue & Indigo & Violet & \\
\hline Pattern & & & & \\
\hline
\end{tabular}

Figure 4 The seven patterns generated by different angles and colors. The order is from left to right, up to down.

\subsection{Installation}

Figure 5 shows the installation for the application which used a projector to project the generated graphics onto the neutral wall, allowing users to sit in front of the projection screen and watch. This allows the user to relax and wear the EEG headset.

\section{USER FEEDBACK}

A user study was conducted over 50 university students, between the ages of $16-25$. Include $44 \%$ of male and $56 \%$ of female. The experiment apparatus and the projector were used to project the patterns to the neutral wall. Each subject used the applications for 2-5 min. After that, subjects were asked to take an online survey. The questions included what subjects thought while using the application and how they felt when they used the applications. Figure 6 shows some patterns made by different subjects.

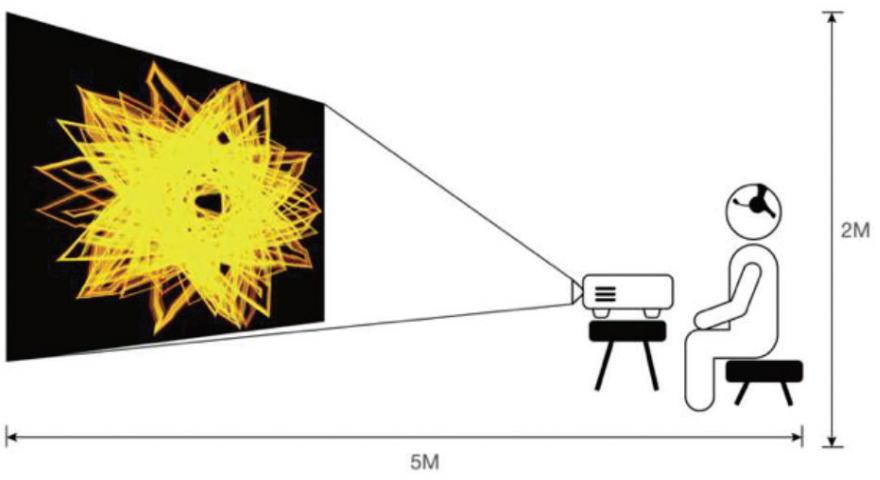

Figure 5 Installation for the application.

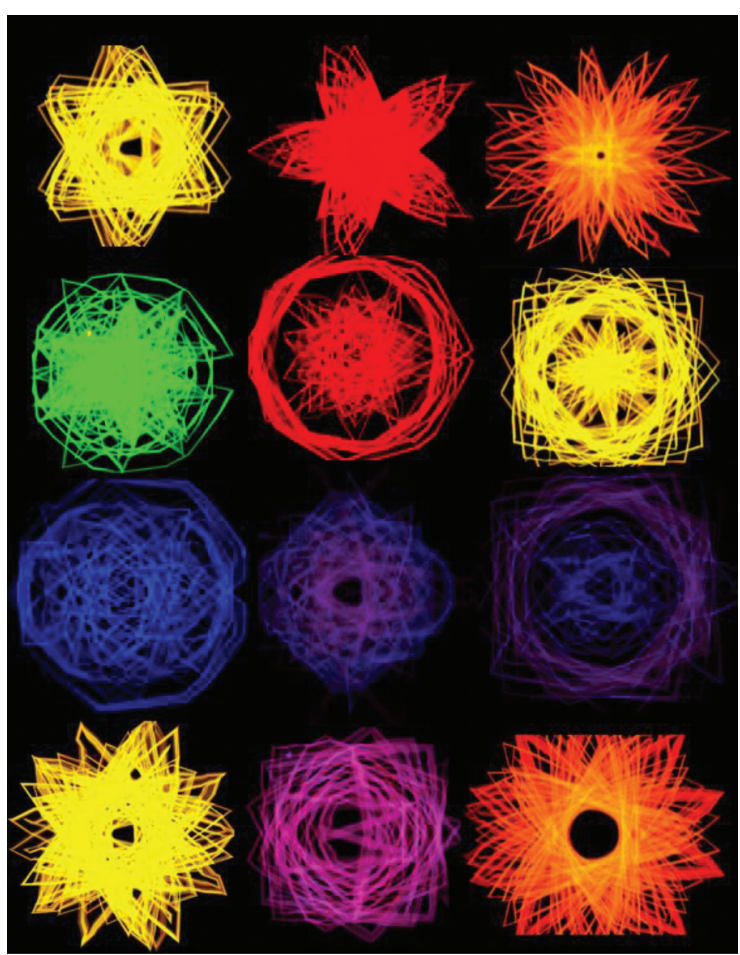

Figure 6 Pattern generated by different subjects. 


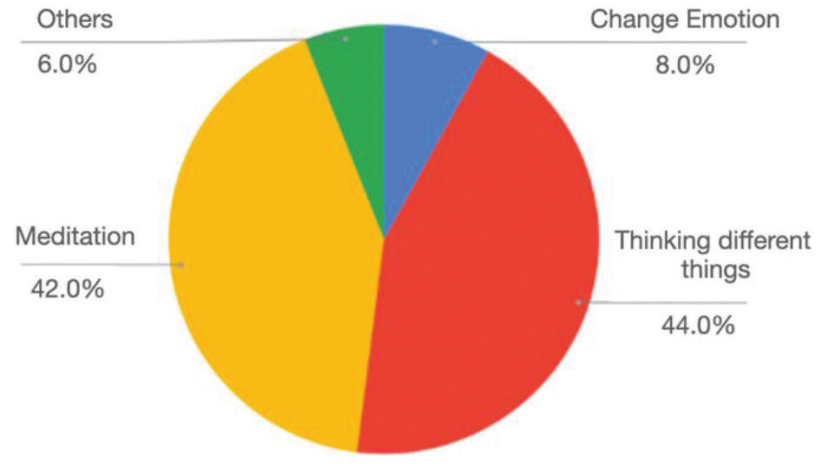

Figure 7 What are people doing while using the application.

Figure 7 shows when people used the application, 44\% tried to think about different things to see the changes in graphics, colors, and sounds. $8 \%$ tried using different emotions to see different changes. $42 \%$ were in the state of emptying meditation. About $6 \%$ were thinking about the meaning of these graphics and how such patterns were made.

Among them, 84\% found it interesting and novel. In addition to the artistry brought about by the graphics and color changes, almost everyone wanted to know the meaning of these graphics and whether they can understand their emotions or psychological state.

\section{CONCLUSION}

The use of EEG to generate phyllotaxis is a very novel experience for most people. As an installation art, the generated graphics have different results according to the conditions of different people, making the installation art show a variety of effects.

Through some applications, users can also recognize their own mental state. Through different thinking patterns, imagination, and memories, people were able to produce graphics of different colors. Seeing and feeling the visual and sound effects of these graphics can trigger new psychological states, such as excitement or calmness. In this way, we have space and time to face our own state of mind and examine our inner state. It is like a dialogue and communication with the self, allowing the heart to settle, reflect, meditate, and think, and find the inner peace and answer.

In further researches, we need to find out what kind of content can make users more relaxed. By setting up different contents and experiential environments, the impact after the subject's daily use could be measured. These experiential findings could be guided to find the method to improve the concentration of the users and how to improve users' creativity and productivity.

\section{CONFLICTS OF INTEREST}

The authors declare they have no conflicts of interest.

\section{ACKNOWLEDGMENT}

This research was supported in part by the Ministry of Science and Technology of Taiwan under the grant (IV) MOST 109-2218-E002-038-.

\section{REFERENCES}

[1] Leap Motion. Accessed December 6, 2020.

[2] Wikipedia, Wii. Accessed December 6, 2020.

[3] Wikipedia, Art therapy. Accessed December 7, 2020.

[4] M. Prpa, P. Pasquier, Brain-computer interfaces in contemporary art: a state of the art and taxonomy, in: A. Nijholt (ed.), Brain Art, Springer, Cham, 2019, pp. 65-115.

[5] M. Zhang, Y. Huang, S.U. Ahmed, M. Shidujaman, Mapping between mind cybernetics and aesthetic structure in real-time EEG art, in: C. Stephanidis, M. Kurosu, H. Degen, L. Reinerman-Jones (eds.), HCI International 2020 - Late Breaking Papers: Multimodality and Intelligence, HCII 2020, Springer, Cham, 2020, pp. 344-362.

[6] NeuroSky, ThinkGear Connector. Accessed December 7, 2020.

[7] Unity. Accessed December 7, 2020.

[8] NeuroSky, Hardware. Accessed March 14, 2021.

[9] A. Lindenmayer, P. Prusinkiewicz, The Algorithmic Beauty of Plants, Springer-Verlag, New York, 1990, pp. 99-109.

[10] NeuroSky, eSenses. Accessed May 4, 2021.

[11] Healthline, chakras. Accessed December 8, 2020.

\section{AUTHORS INTRODUCTION}

\section{Mr. Chien-Tung Lin}

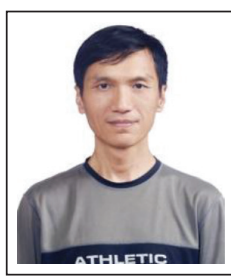

He received his B.S. degree from the Department of Computer Science and Information Management, Providence University, Taiwan in 1999. He is a Graduate student at the Graduate Institute of Animation and Film Art, Tainan National University of the Arts, Taiwan. His research interests include virtual reality, interactive art, EEG, art therapy, computer animation and healing method. 


\section{Dr. R.P.C. Janaka Rajapakse}

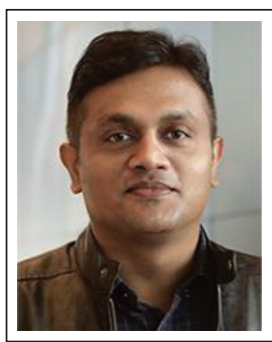

$\mathrm{He}$ is an Associate Professor at the Graduate Institute of Animation and Film Art, Tainan National University of the Arts, Taiwan. And he is also a visiting scholar in the Department of Media and Image Technology at the Faculty of Engineering, Tokyo Polytechnic University, Japan. He was a Postdoctoral Researcher at the Center for Hyper Media Research, Graduate School of Engineering, Tokyo Polytechnic University. He received his $\mathrm{PhD}$ in Knowledge System Science from Japan Advanced Institute of Science and Technology in 2008. His research interests include computer animation, motion capture, VR/AR/MR, haptic interfaces, AI, computer graphics, and Kansei Engineering. He is a member of the Motion Capture Society, The Society for Art and Science, ASIAGRAPH, and SIGDesign Creativity.

\section{Dr. Yoshimasa Tokuyama}

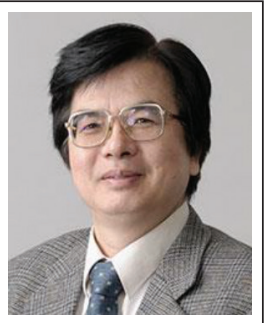

He is a Professor of the Department of Media and Image Technology at the Faculty of Engineering, Tokyo Polytechnic University, Japan. He received his $\mathrm{PhD}$ in Computer Graphics from the University of Tokyo in 2000. His areas of research interest include computer graphics, haptics, virtual reality, shape modeling, game, and their applications. He is a member of the IPSJ, ITE, IIEEJ, and The Society for Art and Science. 